\title{
A Geometric Method for the Numerical Solution of Nonlinear Equations and Its Application to Nonlinear Oscillations
}

\author{
By \\ Yoshitane SHINOHARA*
}

\section{Introduction}

In his previous paper $[1,2]$, the author proposed a new method for the numerical solution of a system of nonlinear equations

$$
\boldsymbol{F}(\boldsymbol{x})=\left\{f_{k}\left(x_{1}, x_{2}, \ldots, x_{m}\right)\right\}=\mathbf{0} \quad(k=1,2, \ldots, m)
$$

in a bounded region. But in these papers he did not describe the techniques of programming for the method proposed.

In the present paper, the techniques of programming will be described and a program written in FORTRAN will be presented. This program is tested on a system consisting of five algebraic equations. Lastly, in illustration, will be shown an application of our program to the computation of subharmonic solutions of Duffing's equation.

The author expresses his hearty gratitude to Professor M. Urabe for his constant advice and encouragement.

The author also wishes to acknowledge the assistance of Miss. T. Takahashi, Mr. Y. Kono and Mr. Y. Nakatani in the computer room of the RIMS, Kyoto University.

Received September 29, 1971 and in revised form February 25, 1972.

Communicated by M. Urabe.

* Faculty of Engineering, Tokushima University, Tokushima 770, Japan. 


\section{The Method of Computation}

We consider a global problem of finding the solutions of the system (E) in a bounded region $R$ :

$$
R=\left\{\left(x_{1}, x_{2}, \ldots, x_{m}\right): l_{i} \leqq x_{i} \leqq m_{i} \quad(i=1,2, \ldots, m)\right\}
$$

We assume that the real-valued functions $f_{k}\left(x_{1}, x_{2}, \ldots, x_{m}\right)(k=1,2, \ldots, m)$ are continuously differentiable in the region $R$. We assume further that the solutions under consideration are all simple, that is, for all solutions of (E) lying in $\boldsymbol{R}$, the Jacobian of $\boldsymbol{F}(\boldsymbol{x})$ with respect to $\boldsymbol{x}$ does not vanish.

From the equations of the system (E), we choose $m-1$ equations

$$
f_{\alpha}\left(x_{1}, x_{2}, \ldots, x_{m}\right)=0 \quad(\alpha=1,2, \ldots, m-1) .
$$

For this system, we request that the rank of the matrix

$$
\left(\partial f_{\alpha} / \partial x_{i}\right) \quad(\alpha=1,2, \ldots, m-1 ; i=1,2, \ldots, m)
$$

is equal to $m-1$. The system of equations (2.1) then determines a curve

$$
C: \boldsymbol{x}=x(s),
$$

for which from (2.1) we have

$$
\sum_{i=1}^{m} \frac{\partial f_{\alpha}}{\partial x_{i}} \cdot \frac{d x_{i}}{d s}=0 \quad(\alpha=1,2, \ldots, m-1)
$$

Put

$$
D_{i}=(-1)^{i} \cdot \frac{\partial\left(f_{1}, f_{2}, \cdots, f_{m-1}\right)}{\partial\left(x_{1}, \ldots, x_{i-1}, x_{i+1}, \ldots, x_{m}\right)} \quad(i=1,2, \ldots, m)
$$

then from (2.2) we have

$$
\frac{d x_{i}}{d s}=\lambda \cdot D_{i} \quad(i=1,2, \ldots, m)
$$

where $\lambda$ is an arbitrary parameter. Let us choose a parameter $s$ so that 
$s$ may be an arc length of the curve $C$. Then we readily have

$$
\lambda= \pm\left[\sum_{i=1}^{m} D_{i}^{2}\right]^{-\frac{1}{2}}
$$

Hence from (2.4), for the curve $C$ we have a system of differential equations of the form

$$
\frac{d \boldsymbol{x}}{d s}=\mathbf{X}(\boldsymbol{x})
$$

Now we take a point $\boldsymbol{x}=\boldsymbol{x}^{(0)}$ on the curve $C$ and suppose $\boldsymbol{x}^{(0)}=\boldsymbol{x}(0)$. Then we can trace the curve $C$ integrating numerically equation (2.6) by a step-by-step method, say, the Runge-Kutta method. Let $x^{(l)}(l=1,2, \ldots)$ be an approximate value of $\boldsymbol{x}(s)$ obtained at the $l$-th step by the numerical integration. Then we may have $f_{m}\left[x^{(0)}\right] \cdot f_{m}\left[x^{(1)}\right] \leqq 0$. Otherwise we continue the numerical integration of (2.6) until we have

$$
f_{m}\left[x^{(l-1)}\right] \cdot f_{m}\left[x^{(l)}\right] \leqq 0
$$

Once we have had (2.7) for some $l$, we check whether $\left|f_{m}\left[x^{(l-1)}\right]\right|$ or $\left|f_{m}\left[\boldsymbol{x}^{(l)}\right]\right|$ is smaller than a specified positive number $\varepsilon$. If this is not satisfied, we multiply the step-size of the numerical integration by $2^{-p}$ $(p \geqq 1)$ and repeat this process. Then after a finite number of repetitions we shall have

$$
\left\{\begin{array}{l}
f_{m}\left[\boldsymbol{x}^{(l-1)}\right] \cdot f_{m}\left[\boldsymbol{x}^{(l)}\right] \leqq 0 \\
\left|f_{m}\left[\boldsymbol{x}^{(l-1)}\right]\right| \text { or }\left|f_{m}\left[\boldsymbol{x}^{(l)}\right]\right|<\varepsilon .
\end{array}\right.
$$

The value $x^{(l-1)}$ or $x^{(l)}$ satisfying (2.8) gives an approximate solution of the given system of equations (E). Starting from $x^{(l-1)}$ or $x^{(l)}$, we then can compute a solution of (E) by the Newton method. However, if $\varepsilon$ is very small, $\boldsymbol{x}^{(l-1)}$ or $\boldsymbol{x}^{(l)}$ itself will give an accurate approximate solution of (E).

Our method is based on the above principle.

In order to find a point $\boldsymbol{x}=\boldsymbol{x}^{(0)}$ on the curve $C$, it suffices to find a solution of the system (2.1) consisting of $m-1$ equations after assigning 
a suitable value to some one of $x_{i}^{9} \mathrm{~s}(i=1,2, \ldots, m)$. Our method is clearly applicable to systems consisting of $m-1$ equations. Hence, repeating such a process, our method is reduced to finding a solution of a single equation.

In the course of the numerical integration of (2.6), it may happen due to the accumulated error that

$$
\left|f_{\alpha}\left[x^{(l)}\right]\right| \geqq \xi
$$

at some $l$-th step for some positive integer $\alpha \leqq m-1$, where $\xi$ is a prescribed positive number. When (2.9) happens, one however can correct $\boldsymbol{x}^{(l)}$ so that the corrected value $\tilde{\boldsymbol{x}}^{(l)}$ may satisfy inequalities

$$
\left|f_{\alpha}\left[\tilde{x}^{(l)}\right]\right|<\xi
$$

for all $\alpha=1,2, \ldots, m-1$. To do so, it suffices to apply the Newton method to (2.1) starting from $x=x^{(l)}$ leaving one of $x_{i}^{(e)}$ 's $(i=1,2, \ldots, m)$ unchanged.

If we continue the numerical integration of (2.6) beyond the approximate solution obtained or begin the numerical integration of (2.6) in the reverse direction, then we shall have (2.8) again provided there are solutions of (E) on the curve. Continuing our process, in the region $R$, we thus can get numerically all solutions of (E) lying on a branch of the curve $C$.

The curve $C$ may consist of some different disconnected branches [Fig. 1, Fig. 2]. In order to find all these branches, a special setup is needed.

We divide each interval $\left[l_{j}, m_{j}\right](j=2,3, \ldots, m)$ into subintervals of equal length $h_{j}$ and denote an arbitrary subinterval obtained by $\left[L_{j}\right.$, $\left.L_{j}+h_{j}\right]$.

To begin with, we consider two equations

$$
\begin{aligned}
& f_{1}\left(x_{1}, x_{2}, l_{3}, L_{4}, \ldots, L_{m}\right)=0, \\
& f_{2}\left(x_{1}, x_{2}, l_{3}, L_{4}, \ldots, L_{m}\right)=0
\end{aligned}
$$


in the region $R_{1}^{[2]}$ :

$$
\begin{gathered}
R_{1}^{\lceil 2]}=\left\{\left(x_{1}, x_{2}, \ldots, x_{m}\right): l_{i} \leqq x_{i} \leqq m_{i}(i=1,2), x_{3}=l_{3},\right. \\
\left.x_{j}=L_{j}(j=4,5, \ldots, m)\right\} .
\end{gathered}
$$

Our method begins with the calculation of the intersections of two plane curves (2.10) and (2.11). In order to find all the branches of the curve (2.10) lying in the region $R_{1}^{[2]}$, we divide the region $R_{1}^{[2]}$ into subregions $D_{i}^{[2]}$ so that

$$
R_{1}^{[2]}=D_{1}^{[2]} \cup D_{2}^{[2]} \cup \cdots \cup D_{n_{2}}^{[2]} \quad \text { [Fig. 1], }
$$

where $D_{i}^{[2]}$ are rectangles with the length $m_{1}-l_{1}$ and the breadth $h_{2}$.

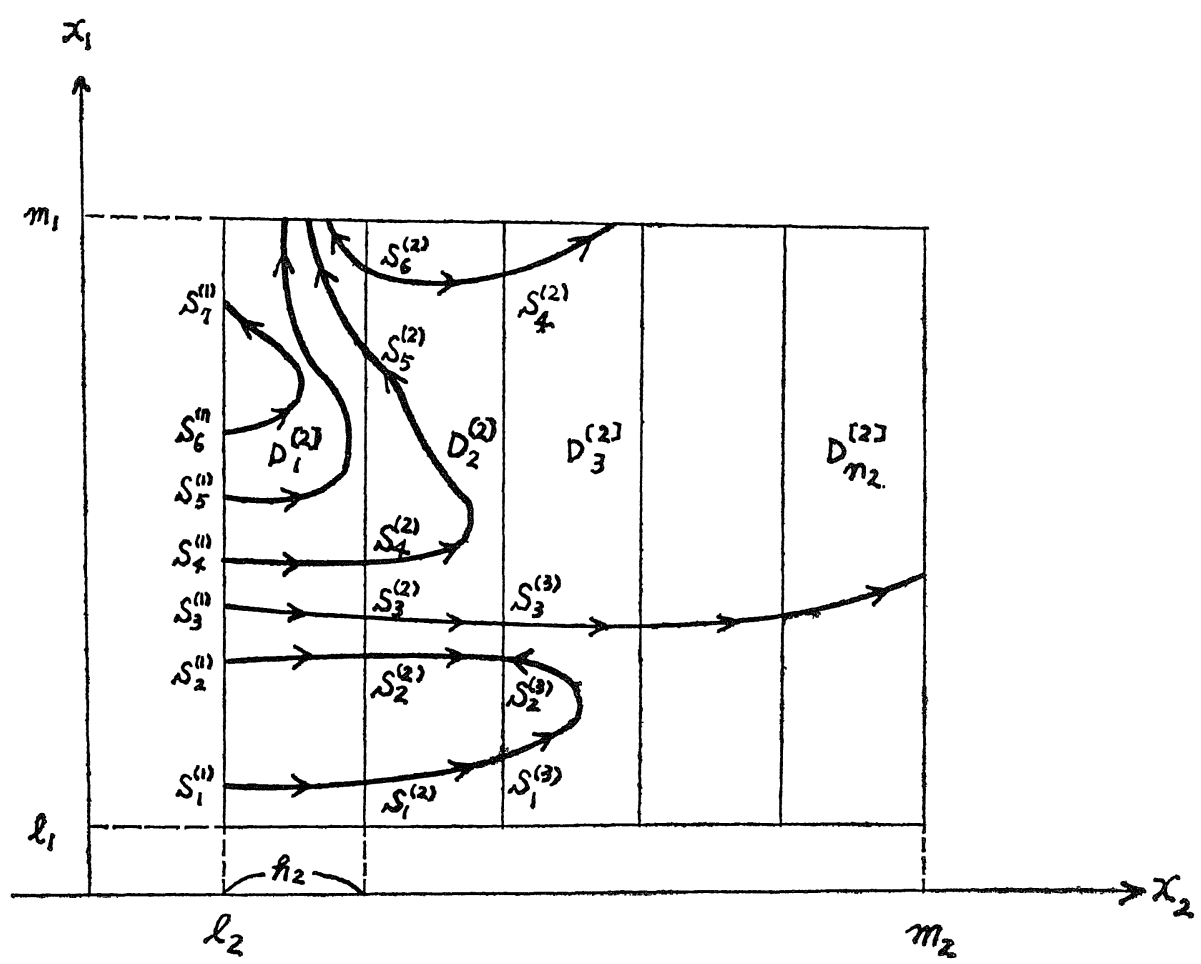

Fig. 1

First, we calculate the intersections $S_{1}^{(1)}, S_{2}^{(1)}, \ldots$ of the curve (2.10) with the straight line $x_{2}=l_{2}$. Starting from these points, we trace the 
branches of the curve (2.10) numerically until we reach the boundary of $D_{1}^{[2]}$. Suppose that the branches of the curve (2.10) have reached the boundary $x_{2}=l_{2}+h_{2}$ of $D_{1}^{[2]}$ at $S_{1}^{(2)}, S_{2}^{(2)}, \ldots$ Then we store these $S_{1}^{(2)}$, $S_{2}^{(2)}, \ldots$ as points of delivery for the succeeding step. In the course of tracing the branches of the curve (2.10), we compute the intersections of these branches with the curve (2.11) with sufficient accuracy.

Next, we calculate the intersections of the curve (2.10) with the straight line $x_{2}=l_{2}+h_{2}$. Some of them are points of delivery obtained at the preceding step. Starting from these points of delivery in the direction of increasing $x_{2}$, we trace the branches of the curve (2.10) numerically until we reach the boundary of $D_{2}^{[2]}$. Suppose that the branches of the curve (2.10) have reached the boundary $x_{2}=l_{2}+2 h_{2}$ of $D_{2}^{[2]}$ at $S_{1}^{(3)}, S_{2}^{(3)}, \ldots$ Then we store these $S_{1}^{(3)}, S_{2}^{(3)}, \ldots$ as points of delivery for the succeeding step. In this tracing, it may happen that the branches starting from the points of delivery on the straight line $x_{2}=l_{2}$ $+h_{2}$ return back to the points, say $S_{5}^{(2)}, \ldots$, on the straight line $x_{2}=l_{2}+h_{2}$ without going outside $D_{2}^{[2]}$. In such a case, the points $S_{5}^{(2)}, \ldots$ may be points of delivery obtained at the preceding step. In such a case the points $S_{5}^{(2)}, \ldots$ are called points of trivial delivery. When the points $S_{5}^{(2)}, \ldots$ are not points of delivery obtained at the preceding step, they are called points of reverse delivery. Clearly points of trivial delivery appear in pairs and we need not trace the branches of the curve (2.10) starting from both points in pairs. As the points of reverse delivery are concerned, it is necessary to trace the branches of the curve (2.10) in the direction of decreasing $x_{2}$ starting from these points. Among the intersections of the curve (2.10) with the straight line $x_{2}=l_{2}+h_{2}$, there may be some points, say $S_{6}^{(2)}, \ldots$, which are neither points of delivery nor points of reverse delivery. When such points appear, it is necessary to trace the branches of the curve (2.10) starting from these points in two directions, that is, the direction of increasing $x_{2}$ and that of decreasing $x_{2}$. When we trace the branches of the curve (2.10) in the direction of increasing $x_{2}$, we may reach the straight line $x_{2}=l_{2}+2 h_{2}$ at some points. In such a case, we store these points as points of delivery for the succeeding step. In the course of tracing the branches of the curve (2.10), we 
always compute the intersections of these branches with the curve (2.11) with sufficient accuracy.

We continue the above process step by step. Then we shall obtain all solutions of the simultaneous equations (2.10) and (2.11) lying in the region $R_{1}^{[2]}=D_{1}^{[2]} \cup D_{2}^{[2]} \cup \cdots \cup D_{n_{2}}^{[2]}$.

Let $S_{1}^{(1)}, S_{2}^{(1)}, S_{3}^{(1)}, S_{4}^{(1)}, \ldots$ be the points corresponding to the solutions of the simultaneous equations (2.10) and (2.11) lying in $R_{1}^{[2] ~(F i g . ~ 2) . ~}$

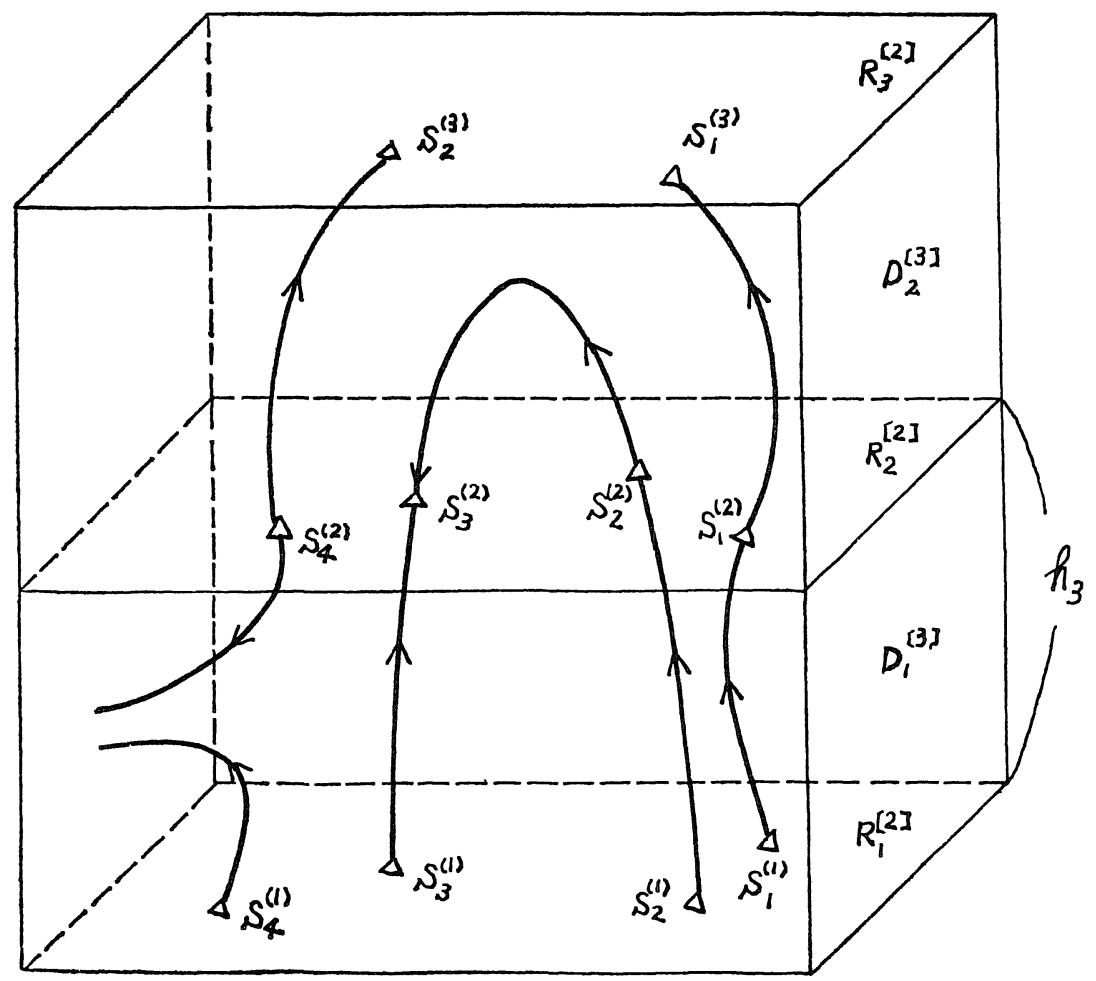

Fig. 2

We consider the three equations

$$
\begin{aligned}
& f_{1}\left(x_{1}, x_{2}, x_{3}, L_{4}, \ldots, L_{m}\right)=0 \\
& f_{2}\left(x_{1}, x_{2}, x_{3}, L_{4}, \ldots, L_{m}\right)=0 \\
& f_{3}\left(x_{1}, x_{2}, x_{3}, L_{4}, \ldots, L_{m}\right)=0
\end{aligned}
$$


in the region $D_{1}^{[3]}$ :

$$
\begin{gathered}
D_{1}^{[3]}=\left\{\left(x_{1}, x_{2}, x_{3}, x_{4}, \ldots, x_{m}\right): l_{i} \leqq x_{i} \leqq m_{i} \quad(i=1,2),\right. \\
\left.l_{3} \leqq x_{3} \leqq l_{3}+h_{3}, x_{j}=L_{j} \quad(j=4,5, \ldots, m)\right\} .
\end{gathered}
$$

Starting from the points $S_{1}^{(1)}, S_{2}^{(1)}, S_{3}^{(1)}, S_{4}^{(1)}, \ldots$, we trace the branches of the curve defined by (2.12) and (2.13) numerically until we reach the boundary of $D_{1}^{[3]}$. If these branches reach the boundary $x_{3}=l_{3}+h_{3}$ of $D_{1}^{[3]}$, say at $S_{1}^{(2)}, S_{2}^{(2)}, S_{3}^{(2)}, \ldots$, then we store these $S_{1}^{(2)}, S_{2}^{(2)}, S_{3}^{(2)}, \ldots$ as points of delivery for the succeeding step. In the course of tracing the branches of the curve defined by (2.12) and (2.13), we compute the intersections of these branches with the surface (2.14) with sufficient accuracy.

Next, by the use of the procedure applied to the simultaneous equations (2.10) and (2.11), we calculate the solutions of the simultaneous equations

$$
\left\{\begin{array}{l}
f_{1}\left(x_{1}, x_{2}, l_{3}+h_{3}, L_{4}, \ldots, L_{m}\right)=0 \\
f_{2}\left(x_{1}, x_{2}, l_{3}+h_{3}, L_{4}, \ldots, L_{m}\right)=0
\end{array}\right.
$$

in the region $R_{2}^{[2]}$ :

$$
\begin{aligned}
R_{2}^{[2]}= & \left\{\left(x_{1}, x_{2}, \ldots, x_{m}\right): l_{i} \leqq x_{i} \leqq m_{i} \quad(i=1,2),\right. \\
& \left.x_{3}=l_{3}+h_{3}, \quad x_{j}=L_{j} \quad(j=4,5, \cdots, m)\right\} .
\end{aligned}
$$

Some of the solutions correspond to points of delivery obtained at the preceding step. Starting from these points of delivery in the direction of increasing $x_{3}$, we trace the branches of the curve defined by (2.12) and (2.13) numerically until we reach the boundary of $D_{2}^{[3]}$ :

$$
\begin{aligned}
D_{2}^{[3]}= & \left\{\left(x_{1}, x_{2}, \ldots, x_{m}\right): l_{i} \leqq x_{i} \leqq m_{i} \quad(i=1,2),\right. \\
& \left.l_{3}+h_{3} \leqq x_{3} \leqq l_{3}+2 h_{3}, \quad x_{j}=L_{j} \quad(j=4,5, \ldots, m)\right\} .
\end{aligned}
$$

If these branches reach the boundary $x_{3}=l_{3}+2 h_{3}$ of $D_{2}^{[3]}$, say at $S_{1}^{(3)}, \ldots$, then we store these $S_{1}^{(3)}, \ldots$ as points of delivery for the succeeding step. 
If the branches starting from the points of delivery on the plane $x_{3}=l_{3}$ $+h_{3}$ return back to the points, say $S_{3}^{(2)}, \ldots$, on the plane $x_{3}=l_{3}+h_{3}$ without going outside $D_{2}^{[3]}$, then the points $S_{3}^{(2)}, \ldots$ are either points of trivial delivery, that is, the points coincident with some points of delivery obtained at the preceding step, or points of reverse delivery, that is, the points which do not coincide with any points of delivery obtained at the preceding step. Clearly points of trivial delivery appear in pairs and we need not trace the branches of the curve defined by (2.12) and (2.13) from both points in pairs. From the points of reverse delivery, however, it is necessary to trace the branches of the curve defined by (2.12) and (2.13) in the direction of decreasing $x_{3}$. Among the points corresponding to the solutions of $(2.15)$, there may be some points, say $S_{4}^{(2)}, \ldots$, which are neither points of delivery nor points of reverse delivery. From these points, if exist, it is necessary to trace the branches of the curve defined by (2.12) and (2.13) in two directions, that is, the direction of increasing $x_{3}$ and that of decreasing $x_{3}$. When we trace the branches of the curve defined by (2.12) and (2.13) in the direction of increasing $x_{3}$, we may reach the plane $x_{3}=l_{3}+2 h_{3}$ at some points. In such a case, we store these points as points of delivery for the succeeding step. In the course of tracing the branches of the curve defined by (2.12) and (2.13), we always compute the intersections of these branches with the surface (2.14) with sufficient accuracy.

We continue the above process step by step. Then we shall obtain all solutions of the simultaneous equations (2.12), (2.13) and (2.14) lying in the region $R_{1}^{[3]}=D_{1}^{[3]} \cup D_{2}^{[3]} \cup \cdots \cup D_{n_{3}}^{[3]}$.

Let $S_{1}^{(1)}, S_{2}^{(1)}, \ldots$ be the points corresponding to the solutions (2.12), (2.13) and (2.14) lying in $R_{1}^{[3]}$. We consider the four equations

$$
\begin{aligned}
& f_{1}\left(x_{1}, x_{2}, x_{3}, x_{4}, L_{5}, \cdots, L_{m}\right)=0, \\
& f_{2}\left(x_{1}, x_{2}, x_{3}, x_{4}, L_{5}, \cdots, L_{m}\right)=0, \\
& f_{3}\left(x_{1}, x_{2}, x_{3}, x_{4}, L_{5}, \cdots, L_{m}\right)=0, \\
& f_{4}\left(x_{1}, x_{2}, x_{3}, x_{4}, L_{5}, \cdots, L_{m}\right)=0
\end{aligned}
$$


in the region $D_{1}^{[4]}$ :

$$
\begin{aligned}
D_{1}^{[4]}= & \left\{\left(x_{1}, x_{2}, \cdots, x_{m}\right): l_{i} \leqq x_{i} \leqq m_{i} \quad(i=1,2,3),\right. \\
& \left.l_{4} \leqq x_{4} \leqq l_{4}+h_{4}, \quad x_{j}=L_{j} \quad(j=5,6, \ldots, m)\right\} .
\end{aligned}
$$

Starting from the points $S_{1}^{(1)}, S_{2}^{(1)}, \ldots$, we trace the branches of the curve defined by (2.16), (2.17) and (2.18) numerically until we reach the boundary of $D_{1}^{[4]}$. Then we repeat the process applied for the simultaneous equations (2.12), (2.13) and (2.14). Then we shall obtain all solutions of the simultaneous equations (2.16), (2.17), (2.18) and (2.19) lying in the region $R_{1}^{[4]}=D_{1}^{[4]} \cup D_{2}^{[4]} \cup D_{3}^{[4]} \cup \cdots \cup D_{n_{4}}^{[4]}$, where

$$
\begin{aligned}
& D_{\bar{k}}^{[4]}=\left\{\left(x_{1}, x_{2}, \ldots, x_{m}\right): l_{i} \leqq x_{i} \leqq m_{i} \quad(i=1,2,3)\right. \\
&\left.l_{4}+(k-1) h_{4} \leqq x_{4} \leqq l_{4}+k h_{4}, \quad x_{j}=L_{j} \quad(j=5,6, \ldots, m)\right\} .
\end{aligned}
$$

Continuing the above process, after a finite number of steps, we obtain all solutions of the given system (E) lying in the region $R=R_{1}^{[m]}$.

\section{Program and Its Test}

We have written a program of our method in FORTRAN and have tested it on a system of algebraic equations which appears in the factorization of a polynomial.

Our system of algebraic equations is connected with the factorization of the polynomial

$$
P(x)=x^{7}+a_{1} x^{6}+a_{2} x^{5}+\cdots+a_{6} x+a_{7}
$$

into

$$
\left(x^{5}+p x^{4}+q x^{3}+r x^{2}+s x+t\right) \cdot\left(x^{2}+l x+m\right) .
$$

Put

$$
\begin{aligned}
P(x)= & \left(x^{5}+p x^{4}+q x^{3}+r x^{2}+s x+t\right) \cdot\left(x^{2}+l x+m\right) \\
& -\left(k_{1} x^{4}+k_{2} x^{3}+k_{3} x^{2}+k_{4} x+k_{5}\right),
\end{aligned}
$$


then comparing the coefficients of powers of $x$ in both sides, we have

$$
\left\{\begin{array}{l}
p+l=a_{1}, \\
q+p l+m=a_{2}, \\
r+q l+p m-k_{1}=a_{3}, \\
s+r l+q m-k_{2}=a_{4}, \\
t+s l+r m-k_{3}=a_{5}, \\
t l+s m-k_{4}=a_{6}, \\
t m-k_{5}=a_{7} .
\end{array}\right.
$$

Eliminating $l$ and $m$ from these equations, we have

$$
\left\{\begin{array}{l}
k_{1}=f_{1}(p, q, \cdots, t)=p^{3}-2 p q+r-a_{1} \cdot\left(p^{2}-q\right)+a_{2} p-a_{3}, \\
k_{2}=f_{2}(p, q, \cdots, t)=p^{2} q-q^{2}-p r+s-a_{1} \cdot(p q-r)+a_{2} q-a_{4}, \\
k_{3}=f_{3}(p, q, \cdots, t)=p^{2} r-p s-q r+t-a_{1} \cdot(p r-s)+a_{2} r-a_{5}, \\
k_{4}=f_{4}(p, q, \cdots, t)=p^{2} s-p t-q s-a_{1} \cdot(p s-t)+a_{2} s-a_{6}, \\
k_{5}=f_{5}(p, q, \cdots, t)=p^{2} t-q t-a_{1} p t+a_{2} t-a_{7} .
\end{array}\right.
$$

Hence we see that $Q(x)=x^{5}+p x^{4}+q x^{3}+r x^{2}+s x+t$ is a factor of the given polynomial $P(x)$ if and only if the coefficients $(p, q, r, s, t)$ of $Q(x)$ satisfy the equations

$$
f_{k}(p, q, r, s, t)=0 \quad(k=1,2,3,4,5) .
$$

We have tested our program on the system of equations (3.2) for the polynomial

$$
P(x)=\left(x^{2}+1\right) \cdot\left(x^{2}+x+1\right) \cdot(x+\alpha) \cdot(x+\beta) \cdot(x+\gamma)
$$

For (3.3),

$$
\left\{\begin{array}{l}
a_{1}=1+A, a_{2}=2+A+B, a_{3}=1+2 A+B+C, \\
a_{4}=1+A+2 B+C, a_{5}=A+B+2 C, a_{6}=B+C, a_{7}=C,
\end{array}\right.
$$

where 


$$
\left\{\begin{array}{l}
A=\alpha+\beta+\gamma, \\
B=\alpha \beta+\beta \gamma+\gamma \alpha, \\
C=\alpha \beta \gamma .
\end{array}\right.
$$

For $\alpha=0.5, \beta=-0.5, \gamma=-1$, we have tested our program. In this case, by (3.5),

$$
A=-1, \quad B=-0.25, \quad C=0.25
$$

therefore, by (3.4), we have

$$
\begin{aligned}
& a_{1}=0, \quad a_{2}=0.75, \quad a_{3}=-1, \quad a_{4}=-0.25 \\
& a_{5}=-0.75, \quad a_{6}=0, \quad a_{7}=0.25
\end{aligned}
$$

Hence, by (3.1), the equations (3.2) become

$$
\left\{\begin{array}{l}
f_{1}(p, q, r, s, t)=p^{3}-2 p q+r+0.75 p+1=0 \\
f_{2}(p, q, r, s, t)=p^{2} q-q^{2}-p r+s+0.75 q+0.25=0 \\
f_{3}(p, q, r, s, t)=p^{2} r-p s-q r+t+0.75 r+0.75=0 \\
f_{4}(p, q, r, s, t)=p^{2} s-p t-q s+0.75 s=0 \\
f_{5}(p, q, r, s, t)=p^{2} t-q t+0.75 t-0.25=0
\end{array}\right.
$$

Now $Q(x)$ is a factor of $P(x)$ given by (3.3). Therefore, if $p, q, r, s$ and $t$ are all real, then $Q(x)$ must be one of the following polynomials:

$$
\begin{aligned}
& \left(x^{2}+1\right) \cdot(x+0.5) \cdot(x-0.5) \cdot(x-1) \\
& \quad=x^{5}-x^{4}+0.75 x^{3}-0.75 x^{2}-0.25 x+0.25 \\
& \left(x^{2}+x+1\right) \cdot(x+0.5) \cdot(x-0.5) \cdot(x-1)=x^{5}-0.25 x^{3}-x^{2}+0.25 \\
& \left(x^{2}+1\right) \cdot\left(x^{2}+x+1\right) \cdot(x+0.5)=x^{5}+1.5 x^{4}+2.5 x^{3}+2 x^{2}+1.5 x+0.5 \\
& \left(x^{2}+1\right) \cdot\left(x^{2}+x+1\right) \cdot(x-0.5)=x^{5}+0.5 x^{4}+1.5 x^{3}+0.5 x-0.5 \\
& \left(x^{2}+1\right) \cdot\left(x^{2}+x+1\right) \cdot(x-1)=x^{5}+x^{3}-x^{2}-1 .
\end{aligned}
$$

This means that the real solutions of equation (3.6) are 


$$
(p, q, r, s, t)=\left\{\begin{array}{l}
(-1,0.75,-0.75,-0.25,0.25) \\
(0,-0.25,-1,0,0.25) \\
(1.5,2.5,2,1.5,0.5) \\
(0.5,1.5,0,0.5,-0.5) \\
(0,1,-1,0,-1)
\end{array}\right.
$$

By the use of our program, we have computed real solutions of the equations (3.6) in the region:

$$
\begin{aligned}
& -1.5 \leqq p \leqq 2, \quad-0.6 \leqq q \leqq 3, \quad-1.5 \leqq r \leqq 2.5 \\
& -0.5 \leqq s \leqq 1.9, \quad-1 \leqq t \leqq 1
\end{aligned}
$$

The result obtained is as follows.

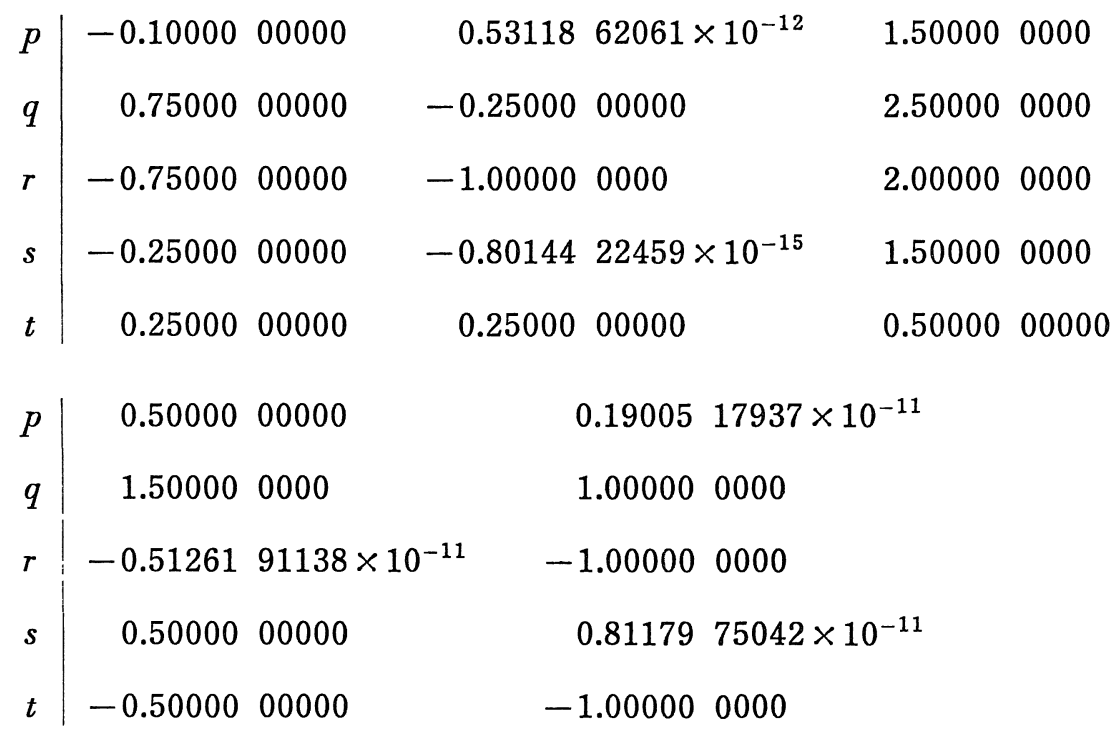

This result shows the usefulness of our method and program. 


\section{Application to the Computation of Subharmonic Solutions of Duffing's Equation}

In papers [3] and [5], M. Urabe has developed Galerkin's procedure for the computation of periodic solutions of nonlinear periodic differential equations, and in paper [4], he has investigated the subharmonic solutions of Duffing's equation by means of the techniques developed by himself in [3] and [5]. In his method, the Newton method is employed for the numerical solution of the determining equation, that is, the equation which should be satisfied by the Fourier coefficients of the trigonometric polynomial approximating the desired periodic solution. In order to find starting approximate solutions of the determining equation, he solves the determining equation consisting of a very small number of equations making use of graphical methods or perturbation techniques. This method of finding starting approximate solutions, however, does not seem to work well always.

In order to find starting approximate solutions, we can apply our method to the determining equation consisting of a moderate number of equations. For experimentation, we have applied our method to the determining equation for $1 / 3$-order subharmonic solutions of Duffing's equation.

We consider Duffing's equation in the form

$$
\frac{d^{2} x}{d t^{2}}+\frac{\sigma}{\omega} \cdot \frac{d x}{d t}+\frac{1}{\Omega} \cdot x\left(1+\varepsilon x^{2}\right)=\frac{1}{\Omega} \cdot \cos t
$$

where $\Omega=\omega^{2}$.

A 1/3-order subharmonic solution of (4.1) is a solution of (4.1) of the form

$$
x(t)=c_{1}+\sum_{n=1}^{\infty}\left(c_{2 n} \cdot \sin \frac{n}{3} t+c_{2 n+1} \cdot \cos \frac{n}{3} t\right) .
$$

Replacing $t$ by $3 t$ in (4.1) and (4.2), we see that a $1 / 3$-order subharmonic solution of (4.1) is a solution of the form 


$$
x(t)=c_{1}+\sum_{n=1}^{\infty}\left(c_{2 n} \cdot \sin n t+c_{2 n+1} \cdot \cos n t\right)
$$

of the equation

$$
\frac{d^{2} x}{d t^{2}}+\frac{3 \sigma}{\omega} \cdot \frac{d x}{d t}+\frac{9}{\Omega} \cdot x\left(1+\varepsilon x^{2}\right)=\frac{9}{\Omega} \cdot \cos 3 t .
$$

If $x(t)$ is a solution of (4.4), then $-x(t+\pi)$ is also a solution of (4.4). Hence we suppose that

$$
-x(t+\pi)=x(t)
$$

is valid for every $1 / 3$-order subharmonic solution (this is certified for subharmonic solutions obtained in [4] by numerical computations). Then for a 1/3-order subharmonic solution, instead of (4.3), we have

$$
x(t)=\sum_{n=1}^{\infty}\left[c_{2 n} \cdot \sin (2 n-1) t+c_{2 n+1} \cdot \cos (2 n-1) t\right] .
$$

In order to find starting approximate solutions, we consider a Galerkin approximation (see [4]) of the form

$$
\bar{x}(t)=c_{2} \cdot \sin t+c_{3} \cdot \cos t+c_{4} \cdot \sin 3 t+c_{5} \cdot \cos 3 t .
$$

By $[4]$, we have the following determining equation for (4.6):

$$
\left\{\begin{aligned}
f_{1}(p, q, r, s)= & \left(\frac{9}{\Omega}-1\right) p-\frac{3 \sigma}{\omega} q+\frac{9 \varepsilon}{\Omega} \cdot\left(0.75 p^{3}-0.75 p^{2} r\right. \\
& \left.+0.75 q^{2} r+0.75 p q^{2}+1.5 p r^{2}+1.5 p s^{2}-1.5 p q s\right)=0 \\
f_{2}(p, q, r, s)= & \frac{3 \sigma}{\omega} \cdot p+\left(\frac{9}{\Omega}-1\right) q+\frac{9 \varepsilon}{\Omega} \cdot\left(0.75 q^{3}+0.75 p^{2} q\right. \\
& \left.-0.75 p^{2} s+0.75 q^{2} s+1.5 q r^{2}+1.5 q s^{2}+1.5 p q r\right)=0 \\
f_{3}(p, q, r, s)= & \left(\frac{9}{\Omega}-9\right) r-\frac{9 \sigma}{\omega} s+\frac{9 \varepsilon}{\Omega} \cdot\left(-0.25 p^{3}+0.75 r^{3}\right. \\
& \left.+1.5 p^{2} r+1.5 q^{2} r+0.75 p q^{2}+0.75 r s^{2}\right)=0 \\
& \left.-0.75 p^{2} q+1.5 p^{2} s+1.5 q^{2} s+0.75 r^{2} s\right)=0
\end{aligned}\right.
$$


where

$$
p=c_{2}, \quad q=c_{3}, \quad r=c_{4}, \quad s=c_{5} .
$$

For

$$
\sigma=2^{-5}, \quad \varepsilon=1, \quad \omega=4,
$$

by the use of our program we have computed solutions of (4.7) in the region:

$$
|p| \leqq 3, \quad|q| \leqq 3, \quad|r| \leqq 0.3, \quad|s| \leqq 0.3
$$

\begin{tabular}{|c|c|c|c|c|c|}
\hline & $p$ & & $q$ & $r$ & $s$ \\
\hline 1: & 0.72425 & 89710 & -0.7325543253 & 0.0152220003 & -0.0602879583 \\
\hline 2: & 0.27228 & 11702 & 0.9935038304 & 0.0152220003 & -0.0602879583 \\
\hline 3: & -0.99654 & 01409 & -0.2609495049 & 0.0152220003 & -0.0602879583 \\
\hline 70 & 0.66808 & 50948 & 0.7162513275 & 0.0142433206 & -0.0845508252 \\
\hline 5. & -0.95433 & 43925 & 0.2204530000 & 0.0142433206 & -0.0845508252 \\
\hline & 0.28624 & 92976 & -0.9367043277 & 0.0142433206 & -0.0845508252 \\
\hline & 0.00000 & 00000 & 0.0000000000 & 0.0005557640 & -0.0666768579 \\
\hline
\end{tabular}

and we have obtained the following solutions.

Table 1

Now from the form of (4.4), we can easily see that if $x(t)$ is a solution of (4.4), then $x[t+(2 \pi / 3)]$ and $x[t+(4 \pi / 3)]$ are also solutions of (4.4), and that if $\bar{x}(t)$ is a Galerkin approximation of a $2 \pi$-periodic solution of (4.4), then $\bar{x}[t+(2 \pi / 3)]$ and $\bar{x}[t+(4 \pi / 3)]$ are also Galerkin approximations of $2 \pi$-periodic solutions of (4.4) with the same order as $\bar{x}(t)$. For the solutions of (4.7) shown in Table 1 , we readily see that the Galerkin approximations $\bar{x}_{2}(t)$ and $\bar{x}_{3}(t)$ corresponding to the 2nd and 3rd solutions in Table 1 are equal respectively to $\bar{x}_{1}[t+(2 \pi / 3)]$ and $\bar{x}_{1}[t+(4 \pi / 3)]$, where $\bar{x}_{1}(t)$ is the Galerkin approximation corresponding to the first solution in Table 1 . Likewise we readily see that the Galerkin 
approximations $\bar{x}_{5}(t)$ and $\bar{x}_{6}(t)$ corresponding to the 5 th and 6 th solutions in Table 1 are equal respectively to $\bar{x}_{4}[t+(2 \pi / 3)]$ and $\bar{x}_{4}[t+(4 \pi / 3)]$, where $\bar{x}_{4}(t)$ is the Galerkin approximation corresponding to the 4 th solution in Table 1 . The Galerkin approximation $\bar{x}_{7}(t)$ corresponding to the 7 th solution in Table 1 will be supposed to be a Galerkin approximation of a harmonic solution of original Duffing's equation (4.1).

Starting from the solutions of (4.7) shown in Table 1, by the use of the techniques described in [4] we have computed Galerkin approximations of higher order for subharmonic solutions and harmonic solution. However, by the reason mentioned above, we have not carried out the computations starting from the 2nd, 3rd, 5th and 6th solutions in Table 1. Tables 2 and 3 show the results. In these tables, for each approximate solution, is given an error bound $\delta$ such that

$$
\left[|\bar{x}(t)-\hat{x}(t)|^{2}+|\dot{\bar{x}}(t)-\dot{\hat{x}}(t)|^{2}\right]^{\frac{1}{2}} \leqq \delta,
$$

where $\cdot=d / d t$ and $\hat{x}(t)$ is an exact solution corresponding to the approximate solution $\bar{x}(t)$.

Periodic solutions of (4.4) with $\sigma=2^{-5}, \varepsilon=1, \omega=4$ :

$$
\begin{aligned}
& 1 \quad \bar{x}_{1}(t)=0.7245614343 \sin t \quad-0.7322200674 \cos t \\
& +0.0152223982 \sin 3 t \quad-0.0603311349 \cos 3 t \\
& +0.0011292234 \sin 5 t \quad+0.0002138735 \cos 5 t \\
& +0.0000331833 \sin 7 t \quad-0.0000000135 \cos 7 t \\
& +0.0000005831 \sin 9 t \quad+0.0000006017 \cos 9 t \\
& +0.0000000138 \sin 11 t+0.0000000272 \cos 11 t \\
& -0.0000000001 \sin 13 t+0.0000000007 \cos 13 t \text {. } \\
& \delta=6.6 \times 10^{-8} \text {, Stability: stable. }
\end{aligned}
$$

$2 \bar{x}_{4}(t)=0.6682585789 \sin t \quad+0.7157829204 \cos t$

$+0.0142401915 \sin 3 t \quad-0.0846509661 \cos 3 t$

$-0.0015434867 \sin 5 t \quad-0.0002897473 \cos 5 t$

$+0.0000233942 \sin 7 t \quad+0.0000735294 \cos 7 t$ 


$$
\begin{gathered}
+0.0000022613 \sin 9 t-0.0000016730 \cos 9 t \\
-0.0000000815 \sin 11 t-0.0000000660 \cos 11 t \\
-0.0000000013 \sin 13 t+0.0000000037 \cos 13 t \\
+0.0000000001 \sin 15 t . \\
\delta=1.3 \times 10^{-7} \text {, Stability: unstable. } \\
\text { Table } 2
\end{gathered}
$$

Periodic solution to (4.1) with $\sigma=2^{-5}, \varepsilon=1, \omega=4$ :

$$
\begin{aligned}
& \bar{x}_{7}(t)=0.0005557640 \sin t \quad-0.0666768581 \cos t \\
& +0.0000000143 \sin 3 t \quad-0.0000005181 \cos 3 t \text {. }
\end{aligned}
$$

$\delta=1.5 \times 10^{-9}$, Stability: stable.

Table 3

\section{References}

[1] Shinohara, Y., A geometric method of numerical solution of nonlinear equations and error estimate by Urabe's proposition, Publ. RIMS. Kyoto Univ. 5 (1969), 1-9.

[2] - The geometric method and a generalized Bairstow method for numerical solution of polynomial equation, J. Math. Tokushima Univ. 4 (1970), 19-32.

[3] Urabe, M., Galerkin's procedure for nonlinear periodic systems, Arch. Rational Mech. Anal. 20 (1965), 120-152.

[4] - Numerical investigation of subharmonic solutions to Duffing's equation, Publ. RIMS. Kyoto Univ. 5 (1969), 79-112.

[5] Urabe, M. and A. Reiter, Numerical computation of nonlinear forced oscillations by Galerkin's procedure, J. Math Appl. 14 (1966), 107-140.

[6] Yamauchi, J., S. Moriguchi and S. Hitotumatu, Denshikeisanki no tame no suchikeisanho 1, Baifukan, 1965 (Japanese). 


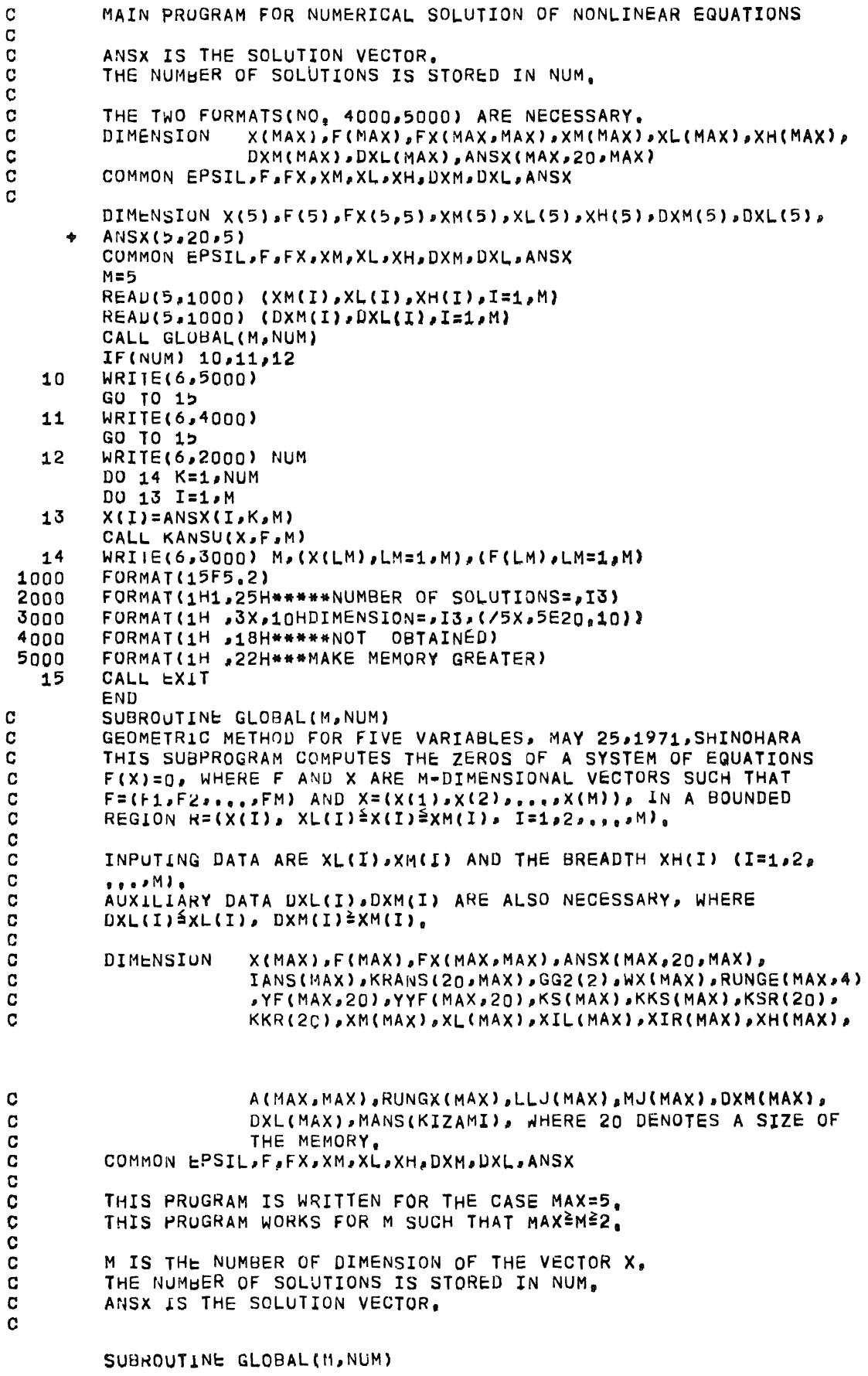


DIMENSIUN $X(5), F(5), F X(5,5), \operatorname{ANS} X(5,20,5)$, IANS $(5), \operatorname{KRANS}(20,5)$,

1 GG2 (2),WX(5),RUNGE $(5,4), Y F(5,20), Y Y F(5,20), K S(5), K K S(5)$,

$K S R(20), \operatorname{KKR}(20), X M(5), X L(5), X I L(5), X I R(5), X H(5), A(5,5), R U N G X(5)$,

$3 \quad L L J(5), M J(5), D X M(5), D X L(5), M A N S(5)$

COMMON EPSIL, $F, F X, X M, X L, X H, U X M, D X L, A N S X$

NUMBER OF BITS OF MANTISSA

MANT $=57$

EPSIL $=0.5 *$ *MANT*16,0

C CONST $=100,0$ EPSIL

MEMURY $\approx 20$

DO $10 \quad I=1.5$

C

$\operatorname{MN}(I)=1$

DOMAIN

WRIIE $(6,1200) \quad(X M(I), X L(I), X H(I), I=1, M)$

WRIIE $(6,1300) \quad(D X M(I), D X L(I), I=1, M)$

DO 999 MCUT $=1, \mathrm{KIZAMI}$

WRITE $(6,1400)$ EPSIL,CONST

DO $11 I=1, M$

$11 M J(I)=(X M(I)-X L(I)) / X H(I)+1,0$

DELTAS $=0.03125$

EH=UELTAS

$A L P H A=0,1 E=04$

$J I G E N=2$

$I$ ANS $(5)=0$

IANS $(4)=0$

FIVE $=$ DIMENSIONAL CASE

$M 5=M J(5)$

$M 4=M J(4)$

$M 3=M J(3)$

$M 2=M J(2)$

DO 500 LL $5=1, M 5$

$L L J(5)=L L 5$

$X I L(5)=X L(5)+F L O A T(L L 5=1) * X H(5)$

$X I R(5)=X I L(5)+X H(5)$

WALPHA $=0,1 \mathrm{E}-04$

$K S(4)=0$

$K K S(5)=0$

$X(5)=X I L(5)$

$W X(5)=X(5)$

RUNGX $(5)=X(5)$

IANS $(3)=0$

C FOUR-UIMENSIONAL CASE

757 DO 400 LL $4=1, M 4$

IF (JIGEN-2) $756,756,755$

$756 \quad L L J(4)=L L 4$

$X I L(4)=X L(4)+F L O A T(L L 4=1) * X H(4)$

$X I R(4)=X I L(4)+X H(4)$

WALPHA $=0,1 E=04$

$K S(3)=0$

$K K S(4)=0$

$X(4)=X I L(4)$

$W \times(4)=X(4)$

RUNGX $(4)=X(4)$

IANS $(2)=0$

c

THREE $\triangle D I M E N$

755 DO 300 LL3 $=1, M 3$

IF (JIGEN-2) 753,753,754

754 IF $(M 402) 218,203.218$

$753 \quad$ LLJ $(3)=L L 3$

$X I L(3)=X L(3)+F L O A T(L L 3-1) * X H(3)$

$X I R(3)=X I L(3)+X H(3)$

WALPHA $=0,1 E-04$

$K S(2)=0$ 


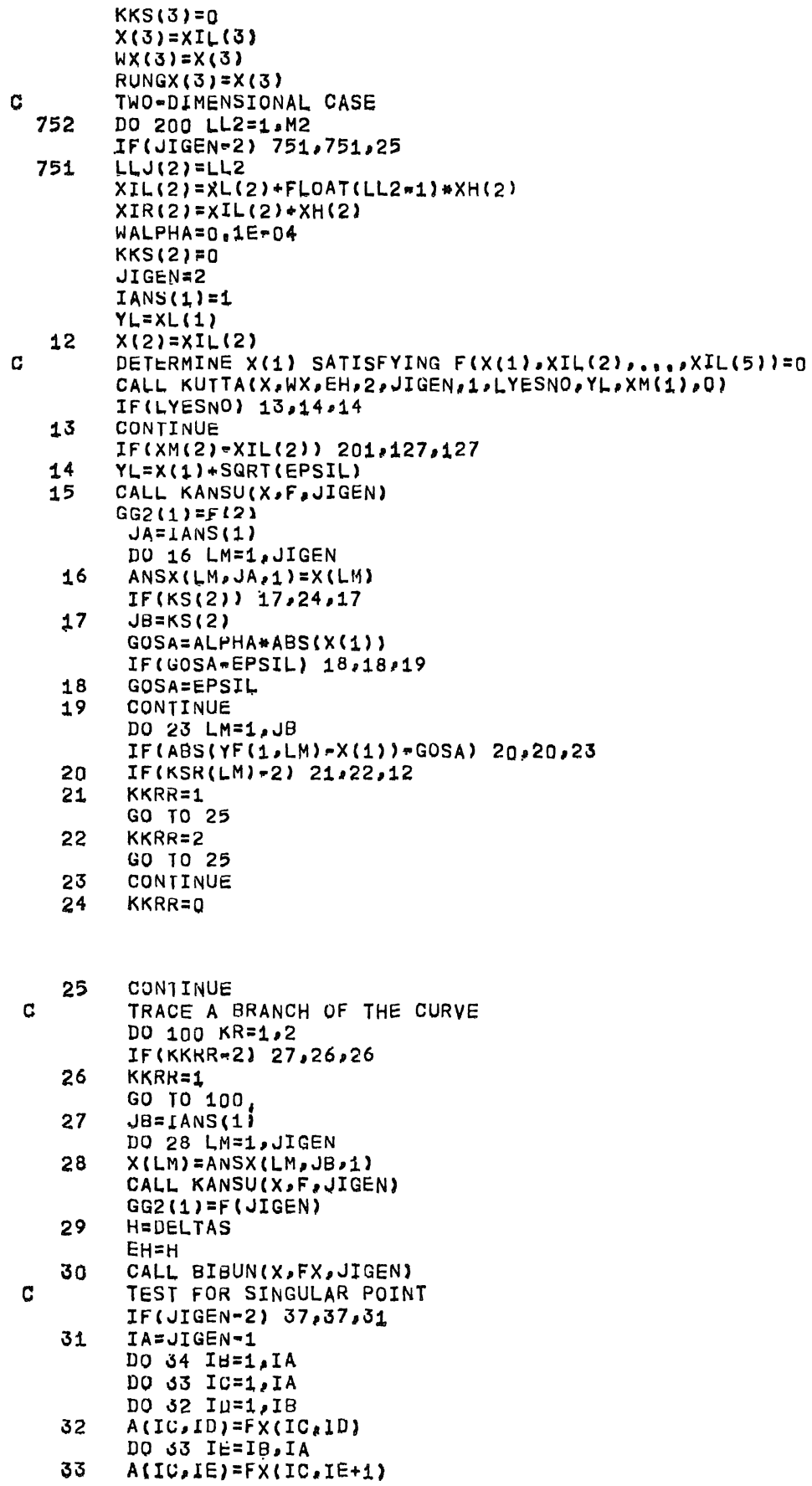


CALL MATINV ( $A, I A, h X, 0, D E T E R M)$

$F(I B)=D E T E R M$

DO 35 IB $=1$, IA

DO 35 IC $=1$, IA

$35 \quad A(I B, I C)=F X(I B, I C)$

CALL MATINV $(A, I A, W X, O, D E T E R M)$

$F(J I G E N)=D E T E R M$

$S \cup M=0,0$

DO 36 IB $=1, J I G E N$

$S U M=S U M+F(I B) * 2$

$S I N G=S Q R T$ (SUM)

GO TO 38

37 SING $=S Q R T(F \times(1,1) * 2+F \times(1,2) * 2)$

38 IF (SING $=$ CONST) $39,40,40$

39 CALL.RANSU $(X, F, M)$

WRITE $(6,2000) \quad$ SING, $(X(I A), F(I A), I A=1, M)$

GO 10123

40 CALL KUTTA (X,WX,EH,KR,JIGEN, $2, L, Y E S N O, Y L, X M(2), 1)$ $J I G=J \perp G E N$

DO 41 IA 1 , JIG

$41 \operatorname{RUNGE}(I A, 1)=W X(I A)$

DO 42 IBE1.JIG

$42 \operatorname{RUNGX}(I B)=X(I B)+\operatorname{RUNGE}(I B, 1) / 2,0$

CALL KUTTA(RUNGX,WX,EH,KR,JIG, $2, L Y E S N O, Y L, X M(2), 1)$

DO $43 \quad I C=1$, JIG

43 RUNGE $(I C, 2)=W X(I C)$

DO 44 ID $=1$, JIG

44 RUNGX (ID) $=X(I D)+R U N G E(I D, 2) / 2,0$

CALL KUTTA(RUNGX,WX,EH,KR,JIG,2,LYESNO,YL,XM(2),1)

DO 45 IE $=1$, JIG

$45 \operatorname{RUNGE}(I t, 3)=W X(I E)$

DO 46 IA $=1, \mathrm{JIG}$

$46 \quad R U N G X(I A)=X(I A)+R U N G E(I A, 3)$

CALL KUTTA (RUNGX,WX,EH,KR,JIG, 2,LYESNO,YL,XM(2),1)

DO 47 I $B=1, J I G$

$47 \operatorname{RUNGE}(I \forall, 4)=W X(I B)$

DO $48 \quad I C=1 . J I G$

$48 W X(I C)=X(I C)+(\operatorname{RUNGE}(I C, 1)+2,0 *(\operatorname{RUNGE}(I C, 2)+R U N G E(I C, 3))$ RUNGE (IC, 4$)) / 6,0$

C STEPESIZE CONTROL

$E M=X H(J I G) * 0.25$

$I F(A B S(W X(J I G)-X(J I G))=E M) \quad 50,49,49$

$49 \quad E H=E H * 0.5$

GO TO 30

50 CALL KANSU $(X, F, J I G E N)$

51 JIH $=J I G E N-1$

$E M=0.1 E-05$

DO b2 IC=1,JIH

$52 E M=A M A X 1(E M, A B S(F(I C))$ )

IF (EM-0.1E-03) 54,53,53

53 CALL NEWTON (JIH,X,KONV,CONST)

IF (KONV) $123,123,30$

54 CALL KANSU(WX, $F, J I G E N)$

DO 55 IC $=1$. JIH

$55 E N=A M A X 1(0.1 E-07, A B S(F(I C)))$

IF (EN-0.1E-02) $56.56,49$

$56 \quad \operatorname{GG} 2(2)=F(J I G)$

$I F(G G 2(1) * G G 2(2)) 96.96 .57$

57 DEX $=W X(J I G)-X I R(J I G)$

RIGHT BUUNDARY TEST

IF (DEX) $\quad 66,66,58$

$58 \quad G O S A=E M * A B S(W X(J I G))$

IF(GOSA-EPSIL) $59.59,60$

59 GUSA $=E P S I L$

60 CONTINUE 
IF (DEX $=G O S A) \quad 64,64,61$

61 IF(JIG-2) 63.63 .62

62 CALL NEWTON (JIH,X,KONV, CONST)

IF (KONV) $123,123,63$

63 IF (EH円EPSIL) $64,64,98$

C

64 JIHIJIGEN

DELIVER THE DATA

WALPHA = AMAX1 (WALPHA,EM)

$\operatorname{KKS}(J I H)=\operatorname{KKS}(J I H)+1$

$J \forall=K K S(J I H)$

65

DO 65 I $B=1, M$

$Y Y F(I B, J B)=W X(I B)$

$K K R(J \forall)=K R$

GO TO 123

c

66 JIH=JIGEN

CHECK WHETHER THE INTEGRAL CURVE RETURNS THE START LINE

SXIL $=(W X(J I H)=X I L(J I H))$ \#(X(JIH) $-X I L(J I H))$

IF (SXIL) 70,67.92

67 IF (JIH-2) $69,69,68$

68 IF (WX(JIH) -XIL(JIH)) $92,80.92$

69

70

71

72

74

75

(1)

$\operatorname{IF}(W X(2)-X I L(2)) \quad 92,74,92$

GOSA $=E M A A B S(X(J I H))$

IF (GOSA-EPSIL) $71,71,72$

GOSA $=E P S I L$

CONIINUE

$\operatorname{IF}(A B S(W X(J I H)-X(J I H))=G O S A) \quad 74,74.73$

IF (EH-EPSIL) 74,74,98

IF $(K S(J I H)) 86,86,75$

$J B=K S(J I H)$

DO 79 LM=1, JB

DO 78 IC $=1, J I H$

GOSA $=A L P H A \# A B S(W X(I C))$

IF (GOSA-EPSIL) $76,76,77$

GOSA $=$ EPSIL

77 CONIINUE

$\operatorname{IF}(A B S(Y F(I C, L M)-W X(I C))-G O S A) \quad 78,78,79$

78 CONIINUE

GO $10 \quad 89$

79 CONTINUE

60 to 86

80 IF(IANS(1)) $123,123,81$

81 JB=IANS (1)

DO $84 \quad L M=1, J B$

DO 84 IC $=1$ JIH

GOSA $=A L P H A * A B S(W X(I C))$

IF (GOSA-EPSIL) $82,82,83$

82 GOSA $=E P S I L$

83 CUNTINUE

IF (ABS (ANSX(IC,LM,1)-WX(IC))-GOSA) $85,85,84$

84 CONTINUE

GO 70123

$85 \operatorname{KRANS}(L M \cdot 1)=3$

GO 10123

86 JIS = JIGEN

$K S(J I S)=K S(J I S)+1$

$\mathrm{JB}=\mathrm{KS}(\mathrm{J} \perp \mathrm{S})$

IF (JB-MEMORY) $866,866,112$

866 DO 87 IH $=1, M$

$87 \quad Y F(I B, J B)=W X(I B)$

$K S R(J \forall)=K R$

IF (JIGEN-2) $\quad 123,123,88$

$88 \quad H=D E L T A S$

$\mathrm{EH}=\mathrm{H}$

GO TO 92

$89 \operatorname{IF}(K S R(L M)) \quad 90,91,90$ 


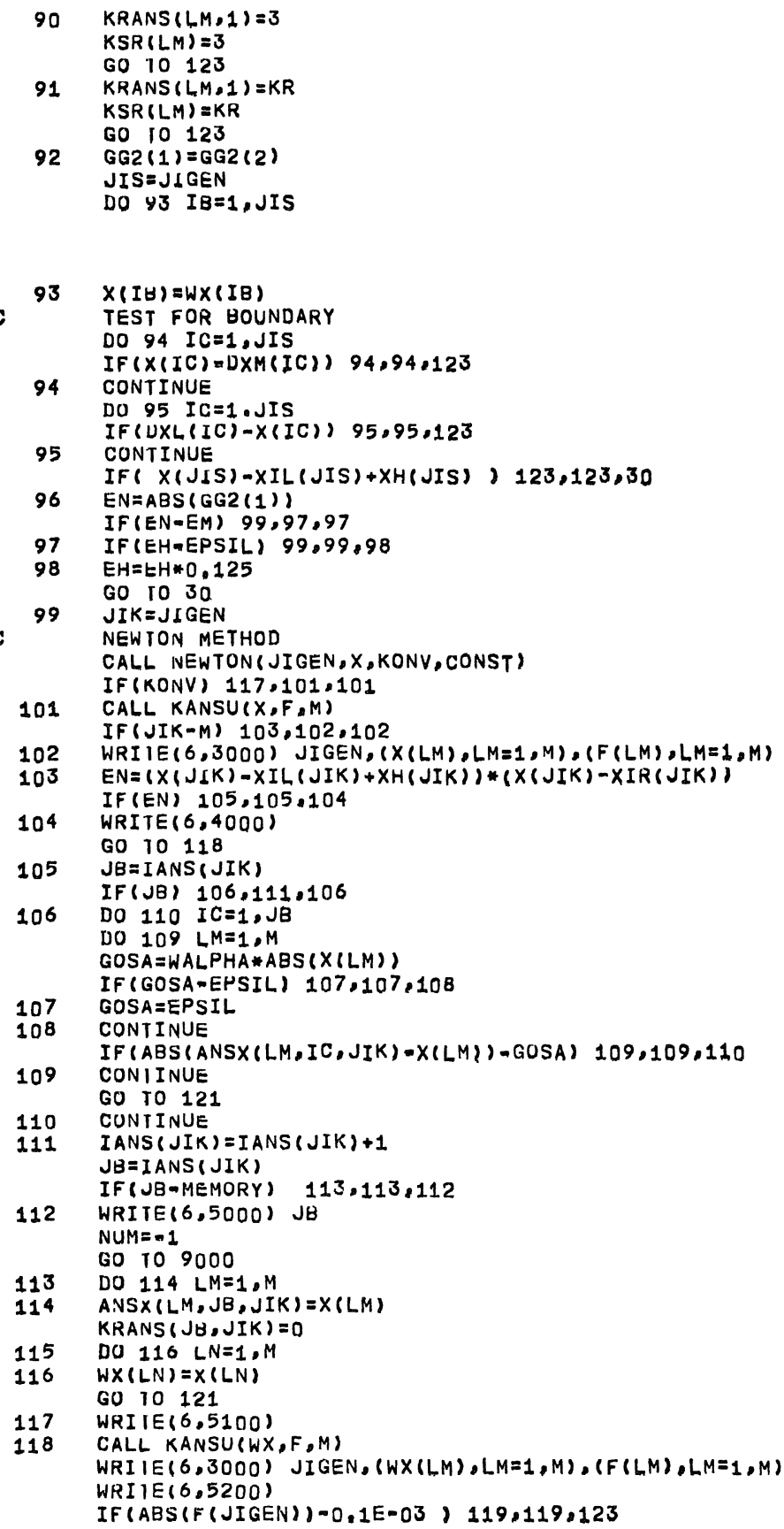




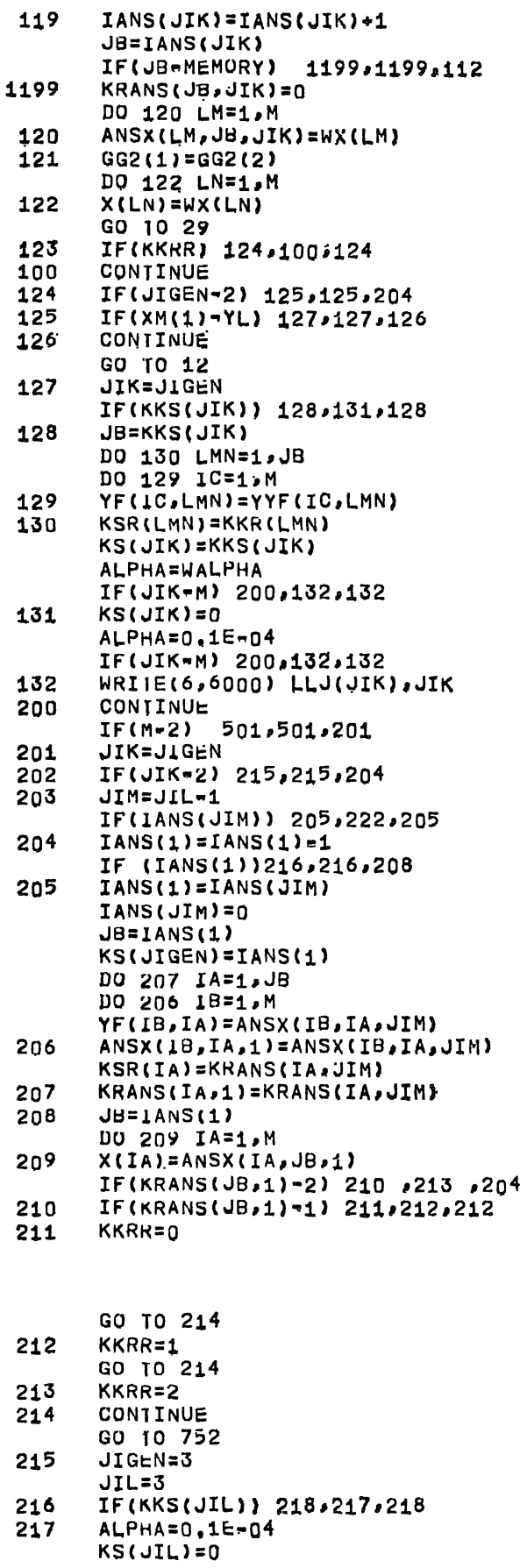




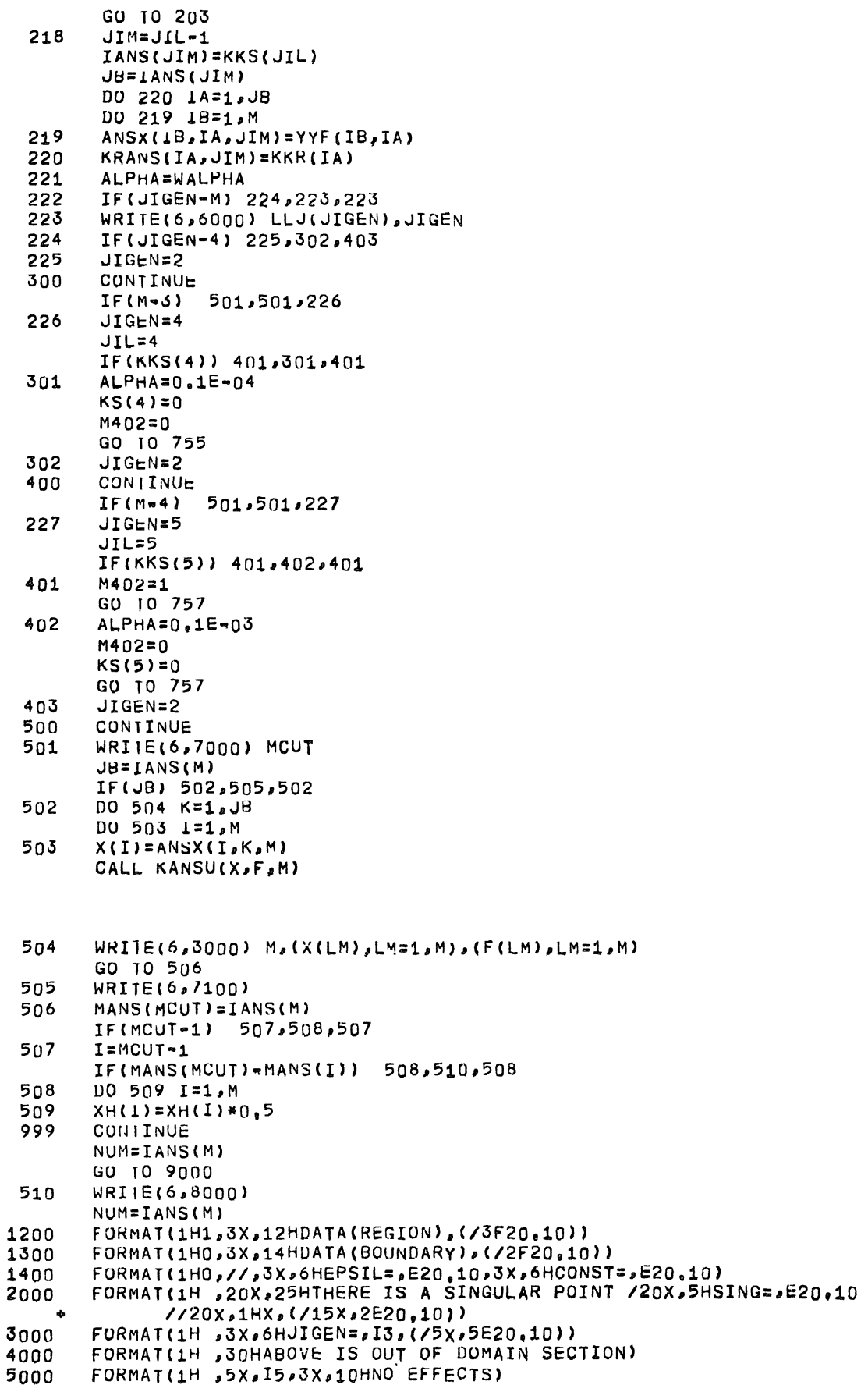




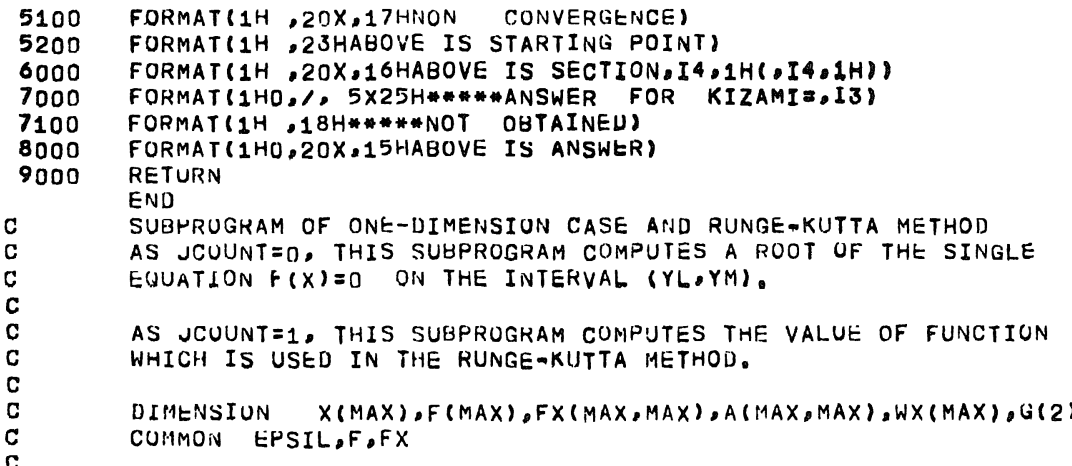

SUBROUTINE KUTTA ( $X, W X, E H, K R, J I G E N, J, L Y E S N O, Y L, Y M, J C O U N T)$

DIMEHSIUN $X(5), F(5), F X(5,5), A(5,5), W X(5), G(2)$

CUMMON EPSIL,F,FX

IF ( JUOUNT-1) 100.200 .200

$E P S=0,1=-04$

DO 40 I $B=1,3$

$D E L, I A G=0.03125$

$X(J)=Y L$

CALL KANSU $(X, F, 1)$

$G(1)=F(1)$

DO 30 IC $=1,8$

$1 \quad X(J)=X(J)+D E L T A G$

CALL $K A N S U(X, F, 1)$

$G(2)=F(1)$

IF (G(1)*G(2), $3,3,2$

$2 \quad G(1)=G(2)$

IF $(Y M-X(J)), 6,1,1$

3 If (ABS(G(1))-EPS) $5,4,4$

4 GUSA $=A B S(X(J))$ \#EPSIL

IF (GOSA-EPSIL) $25,23,24$

GOSA $=E P S I L$

If (ABS (UELTAG) -GOSA) $5,5,25$

$x(J)=x(J)-D E L T A G$

DEL $\mid A G=U E L T A G * 0,125$

IF (ABS (UELTAG)-GOSA) $5,30,30$

30 CUNIINUE

$E P S=E P S * 10.0$

40 CONIINUE

GO 106

5 LYESINU $=1$

GO 107

LYESHU $=-1$

RETURIN

200 I A $=J I G E N-1$

CALL BIBUN (X,FX,IA)

DO 13 IB $=1$, IA

DU 12 IC $=1, I A$

DO $10 I U=1, I B$

$10 \quad A(I C, I D)=F X(I C, I D)$

DO 11 IE $=I B, I A$

$11 \quad A(I C, I E)=F X(I C, I E+1)$

12 CONIINUE

IF $(I A-1) \quad 20,20,21$

$20 \quad F(1)=F \times(1,2)$

$F(2)=F \times(1,1)$

GO 1022

21 CALL MAIINV(A,IA,WX,D,DETERM)

$13 \quad F(I \forall)=D E T E R M$

DU 15 IB $=1$, IA 
DO 14 IC $=1$, IA

$14 \quad A(I \forall, I C)=F X(I B, I C)$

15 CONIINUE

CALL MATINV (A,IA,WX,O,DETERM)

$F(J I G E N)=D E T E R M$

22

16

17

18

19

C
C
C
C

10

17

11

11

12

15

20

SUM $=0,0$

DO 16 IB $=1$, JIGEN

$S: M M=S U M+F(I B) * 2$

SUM $=S Q R I$ ( SUM)

IF $(K K-2) 18,17,17$

SU:M $=-$ SUM

DO 19 IE $=1, J I G E N$

$W X(\perp E)=F(I E) * E H / S U M *(-1,0) * * I E$

RETURN

END

NEWION METHOD

SUBROUT \&NE NEWTON (JIGEN, $X, K O N V$, CONST)

DIMENSIUN $X(M A X), F(M A X), F X(M A X, M A X)$

COMMON EPSIL, F, FX

DIMENSIUN $X(5), F(5), F X(5,5)$

CUMMON EPSIL, $F, F X$

EPS $=$ CUNST

DO 20 KCOUNT $=1.2$

DO 15 I TERA $=1,20$

CALL KANSU $(X, F, J I G E N)$

CALL $\triangle I B U N(X, F X, J I G E N)$

DO $10 I=1$, JIGEN

$F(I)=-F(I)$

CALL MATINV (FX,JIGEN,F, 1, DETERM)

DO $11 \mathrm{~J}=1$, JIGEN

GOSA $=$ EPS $* A B S(X(J))$

IF (GOSA-EPSIL) $16,17,17$

GOSA $=$ PSIL

CONIINUE

IF( $A B S(F(J))-G O S A) \quad 11,11,12$

CONIINUE

GO 1014

DO $13 \mathrm{~J}=1$, JIGEN

$x(J)=X(J)+F(J)$

CONIINUE

EPS $=E P S * 10,0$

CONTINUE

KUNV $=-1$

RETURN

KONV $=1$

RETURN

C
c
c
C
C
C
c
c
c
C

MATRIX COMPUTATIO:V

MATINV ( A, N,B,M,DETERM)

GAUSS JURDAN METHOD.

AS $M=0$. THE DETER:AINANT OF HATRIX A OF ORDER N.IS PLACED IN

DETERM AND NO SIMULTANEOUS SOLUTIDIS ARE CALLED FOR.

AS M=1, THE VECTOR B CONTAINS THE CONSTANT VECTOR WHEN MATINV. IS

CALLED , AND THIS IS REPLACED WITH SOLUTION VECTOR .

DIMENSIUN IPIVOT (MAX), A(MAX,MAX),B(MAX), INDEX(MAX,2),PIVOT(MAX) COMMON EPSIL

SUBROUTINE MATINV $(A, N, B ; M, D E T E R M)$ 


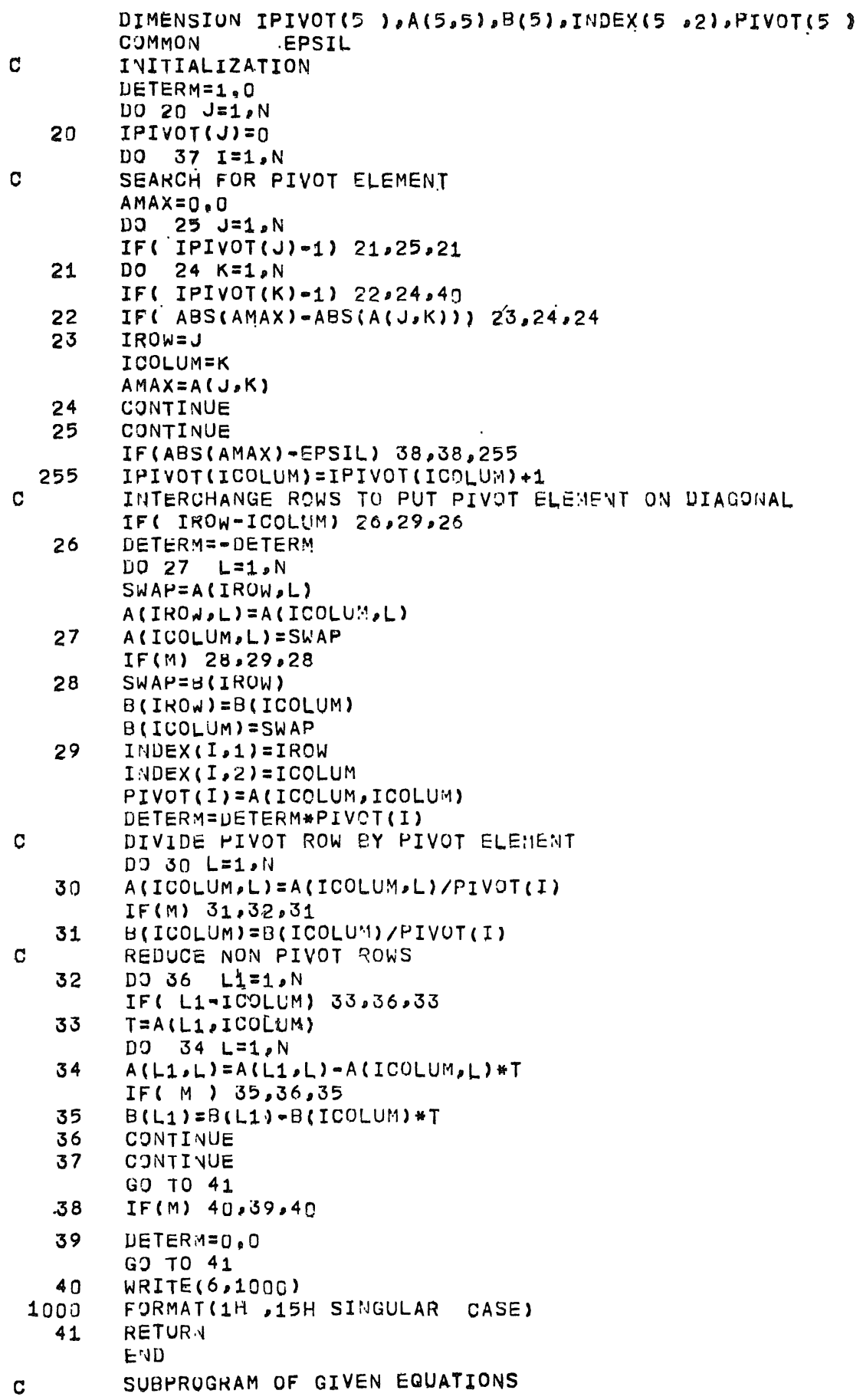


SUBROUTINE KANSU $(X, F, K O S U)$ DIMENSIUN $X(5), F(5)$

$P=X(1)$

$Q=X(2)$

$R=X(3)$

$S=X(4)$

$T=X(5)$

$F(1)=P * \#=2,0 * P * Q+0,75 * P+R+1,0$

IF $(K O S U-2) 20,12,12$

$12 \quad F(2)=Q * P * 2-Q * 2+0,75 * Q-P * R+S+0.25$

IF $(K O S U-3) 20.13 .13$

$13 \quad F(3)=R * P * 2-Q * R+0.75 * R-P * S+T+0.75$

IF (KOSU. 4$) 20.14,14$

$14 \quad F(4)=S * P * 2=Q * S+0.75 * S=P * T$

IF (KOSU-5) 20.15.15

$15 \quad F(5)=T * P * 2-Q * T+0.75 * T=0.25$

20 RETURN

END

C SUBROUTINE OF DERIVATIVES

SUBROUTINE BIBUN $(X, F X, K U S U)$

DIMENSIUN $X(5), F X(5,5)$

$P=X(1)$

$Q=x(2)$

$R=X(3)$

$S=X(4)$

$T=X(5)$

$F X(1,1)=3,0 * P * \# 2-2,0 * 0 * 0,75$

$F X(1,2)=-2,0 * P$

$F X(1,3)=1.0$

$F X(1,4)=0.0$

$F X(1,5)=0,0$

IF (KOSU-2) 20,12,12

$12 \quad F X(2,1)=2.0 * P *(1-K$

$F X(2,2)=P * 2-2,0 * 3+0,75$

$F X(2,3)=-P$

$F X(2,4)=1,0$

$F \times(2,5)=0.0$

IF (KOSU-3) 20.13.13

$13 \quad F X(3,1)=2.0 * P * R=S$

$F X(3,2)=-R$

$F X(3,5)=P * \# 2-Q+0.75$

$F \times(3,4)=-P$

$F X(3, b)=1,0$

IF (KOSU =4) $20,14,14$

$14 F \times(4,1)=2.0 * P * S-T$

$F X(4,2)=-S$

$F X(4,3)=0.0$

$F X(4,4)=P * * 2-Q * 0.75$

$F X(4,5)=-P$

IF (KOSU-5) $20.15 \cdot 15$

$15 \quad F \times(5,1)=2.0 * P * T$

$F X(5,2)=-T$

$F \times(5,3)=0.0$

$F \times(5.4)=0.0$

$F X(5,5)=P * \# 2=Q+0.75$

20

RETURN

END 